\title{
Cryogenic optomechanic cavity in low mechanical loss material
}

\author{
Jérémy Bon, ${ }^{1, a)}$ Leonhard Neuhaus, ${ }^{2, b)}$ Samuel Deléglise, ${ }^{2, c)}$ Tristan Briant, ${ }^{2}$ \\ Philippe Abbé, ${ }^{1}$ Pierre-François Cohadon, ${ }^{2, d)}$ and Serge Galliou ${ }^{1, e)}$ \\ ${ }^{1}$ FEMTO-ST, Univ. Bourgogne Franche-Comté, CNRS, ENSMM, 26 rue de l'Épitaphe, 25030 Besançon, \\ France \\ ${ }^{2}$ Laboratoire Kastler Brossel, UPMC-Sorbonne Universités, CNRS, ENS-PSL Research University, \\ Collège de France, 75005 Paris, France
}

(Received 29 May 2018; accepted 22 July 2018; published online 17 August 2018)

\begin{abstract}
Mechanical losses lower than $10^{-9}$ have been demonstrated on quartz crystal acoustic cavities at liquid helium temperature. In addition, such cavities could rather easily be adapted to be used as optical cavities. This paper addresses the optomechanic devices that could merge from, while highlighting the major assets of each option and showing some preliminary results, including tests on quartz and $\mathrm{CaF}_{2}$ samples. It is shown that the mechanical displacement can be actuated optically by radiation pressure according to proper designs. Published by AIP Publishing.

https://doi.org/10.1063/1.5042058
\end{abstract}

\section{INTRODUCTION}

Bulk acoustic wave (BAW) cavities in quartz crystals can exhibit very low mechanical losses at liquid helium temperature if swept premium quality synthetic crystals are used and the acoustic energy is properly trapped by the cavity geometry. ${ }^{1,2}$ In addition to the easy availability of very pure synthetic quartz crystals, its piezoelectricity offers a convenient way to couple electronics and mechanics. Quartz is thus an ideal candidate to explore the use of optomechanics ${ }^{3}$ to extend traditional applications of quartz BAW cavities to non-piezoelectric but possibly lower-mechanical-loss materials such as calcium fluoride $\left(\mathrm{CaF}_{2}\right)$, sapphire, and diamond. In this scenario, the mechanical modes of a BAW resonator are coupled to an optical field by reflecting a laser beam off a mirror coated on the low-mechanical-loss substrate. This allows low-noise measurement and actuation with free-space optics and without cables or electrodes. However, for technically convenient laser powers, the radiation pressure provided by a single reflection is too weak to significantly drive the motion of a typical BAW mode, and an optical cavity formed by two mirrors is necessary to enhance the interaction between the light and the mirrors. When the laser frequency is tuned to an optical cavity resonance, light is trapped between the two mirrors and the mean intracavity photon flux $I=\frac{2}{\pi} \mathcal{F} I^{i n}$, where $I^{\text {in }}$ denotes the mean incident flux and $\mathcal{F}$ is the finesse of the optical cavity. The finesse can be seen as a measure for the typical number of round trips of light between the two mirrors, and can become very large, i.e., $\mathcal{F} \approx 2 \pi / \mathcal{L}$, provided that the total round-trip loss of the cavity $\mathcal{L}$, i.e., the sum of the transmissions and optical losses of both mirrors, is small compared to one. ${ }^{4}$ With a sufficiently large finesse, such high-Q acoustic cavities would enable the combination of the most coherent mechanical

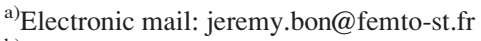

b)Electronic mail: neuhaus@lkb.upmc.fr

${ }^{c)}$ Electronic mail: deleglise@lkb.upmc.fr

d)Electronic mail: cohadon@lkb.upmc.fr

e)Electronic mail: serge.galliou@femto-st.fr
}

system known to date with quantum-limited optical readout and actuation, yielding a very attractive system for various experiments in fundamental physics, ${ }^{5}$ as well as for hybrid quantum systems ${ }^{6}$ and optomechanics. ${ }^{3}$

This paper explores different strategies to construct optomechanical devices from BAW cavities. In particular, we will focus on the canonical choice to co-localize an optical and a mechanical quasi-Gaussian mode along the BAW cavity axis by coating optical mirrors on both sides of the substrate. The tested devices are first described according to their original use, as acoustic cavities. Then, they are examined as optical cavities before finally merging both points of view in various optomechanical systems.

\section{CRYOGENIC QUARTZ ACOUSTIC CAVITIES: FEATURES}

Usual trapped-energy BAW quartz devices have the shape of plano-convex lenses (see Fig. 1). Like in any anisotropic material, three thickness vibration modes can propagate along the normal axis, $y$ often denoted 2, of the lens plane: both fast and slow (quasi-)shear modes, usually called $\mathrm{B}$ and $\mathrm{C}$ modes, respectively, and a (quasi-)longitudinal one, called the A-mode. The A-mode usually exhibits the highest quality factors with values up to several billion, ${ }^{2}$ and will be the only one considered hereinafter in the context of optomechanics. Figure 2 shows typical quality factors measured at $4 \mathrm{~K}$ for a set of doubly rotated $S C$-cut quartz resonators, with $S C$ referring to Stress-Compensated. It might remind that losses in BAW cavities are obviously the sum of losses coming from various origins including engineering losses and intrinsic ones. The engineering process developed for stateof-the-art quartz crystal devices during tens of years reduces the former in such a way that they can become negligible in comparison with the latter: two-level-system losses due to impurities are avoided by sweeping the crystal, surface scattering is rejected by chemical-mechanical polishing, and losses through holders are minimized by using a monolithic structure with an isolated rim and also mainly by trapping 


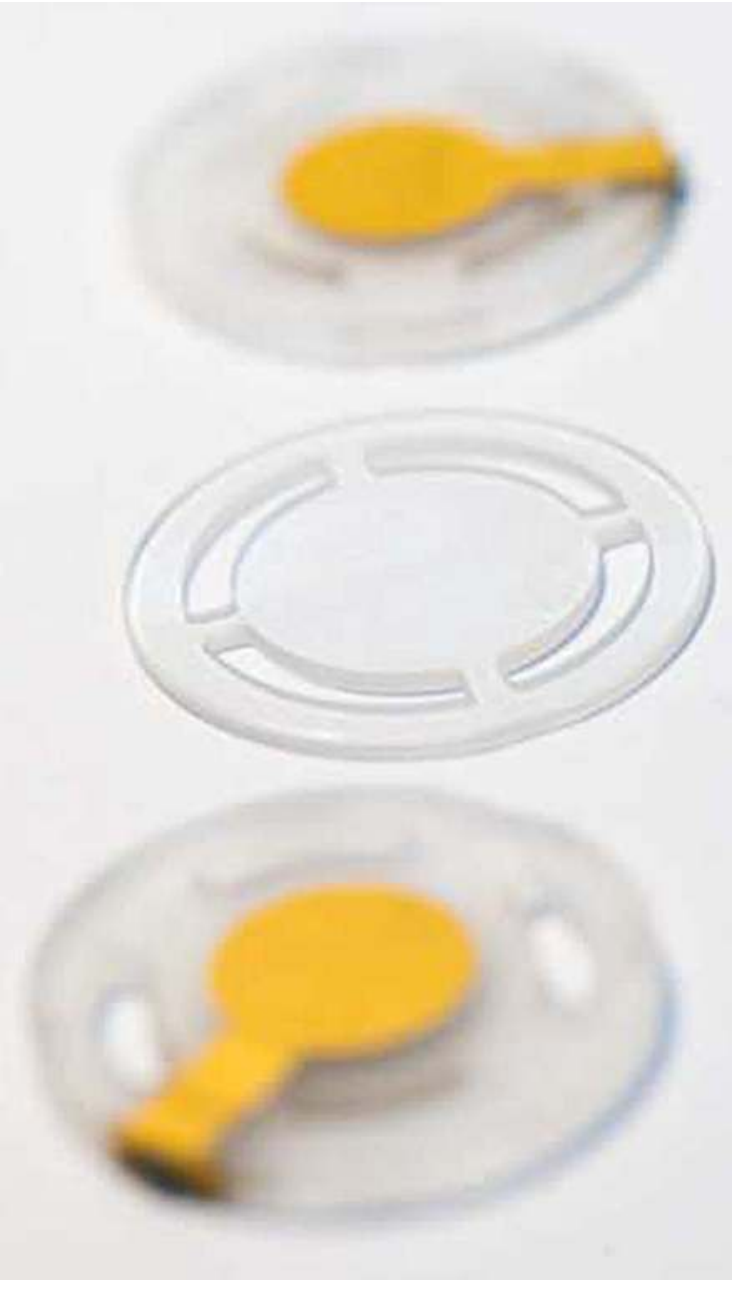

FIG. 1. Quartz crystal resonator with electrode holders (Photo: L. Godard, UFC Besançon, France). The plano-convex BAW cavity located in the center of the structure, in the middle of the photography, is partly isolated from external mechanical disturbances by means of four "bridges" linking the active part to its rim. The two metalized quartz parts at the top and bottom of the photography are optional actuating electrodes which can be placed at a distance of about $1 \mu \mathrm{m}$ on both sides of the BAW cavity when the central structure is sandwiched between these electrode holders. Alternatively, the BAW cavity part can be metallized itself to form an optical cavity colocalized with the mechanical one.

the acoustic energy. Among the remaining intrinsic losses, it can be shown that the thermoelastic ones-irrelevant for shear waves but which might exist for longitudinal waves-become negligible at the operating frequencies, $f>5 \mathrm{MHz}$. As a consequence, the dominant remaining losses come from the interaction of the acoustic wave with thermal phonons. At room temperature, an acoustic resonator vibrating on a mechanical mode at a frequency $f$ exhibits a quality-factor $Q$, i.e., the inverse of losses, limited by $Q \times f=$ constant $\approx 10^{13} \mathrm{~Hz}$. This relationship is derived from the Akheiser regime. ${ }^{7}$ When the temperature goes down below $10 \mathrm{~K}$, mechanisms of phononphonon interactions are different from those at $300 \mathrm{~K}$ because of the increase in the thermal phonon lifetime $\left(2 \pi f \tau_{p h} \gg 1\right.$, where $\tau_{p h}$ is the thermal phonon lifetime): the Q behavior is changed and theoretically does not depend on $f$ anymore, according to the Landau-Rumer theory predicting that the absorption coefficient $\alpha(f)$ is proportional to $f$, more precisely $\alpha(f)$ is proportional to $T^{4} \times f$, with $T$ being the temperature. ${ }^{8,9}$
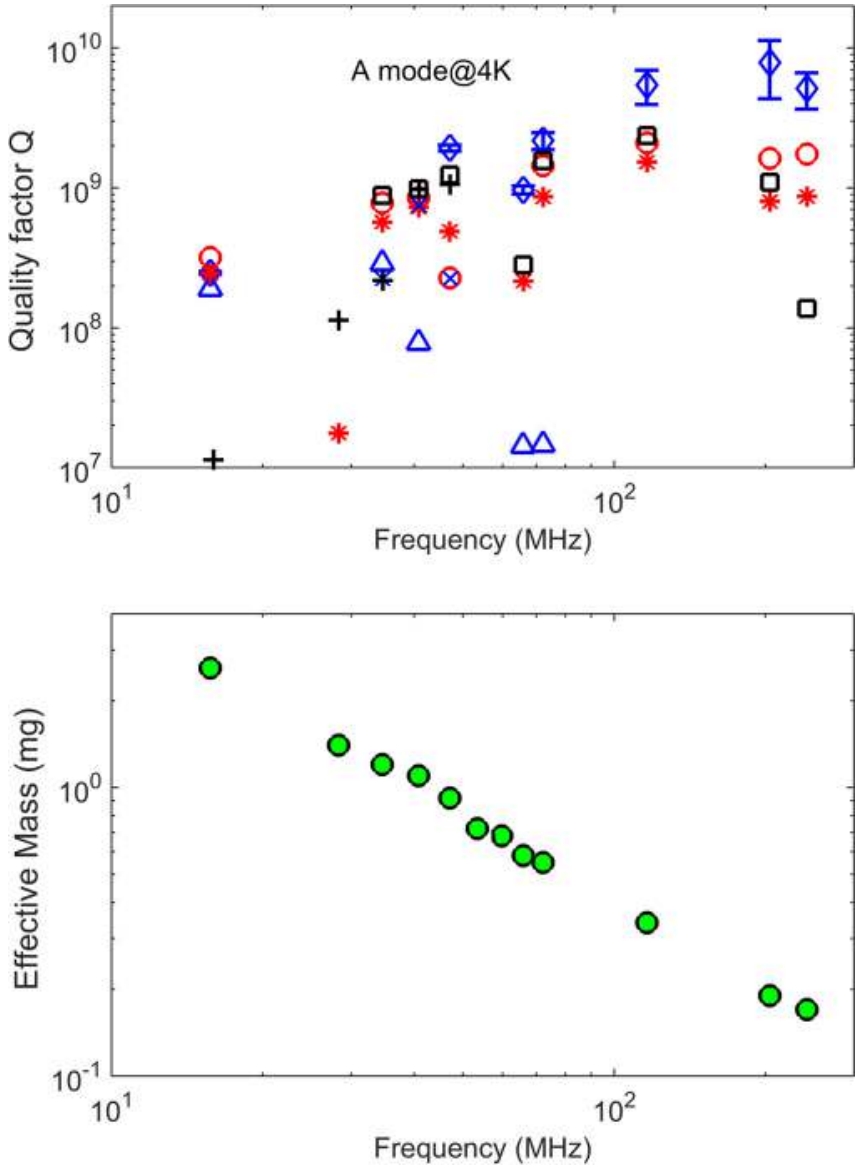

FIG. 2. Top: quality factors versus $n 00$ eigenfrequencies of the thickness (quasi-)longitudinal A-mode of a set of 7 quartz plano-convex acoustic cavities at $4 \mathrm{~K}$. The resonators are $S C$-cuts, which is likely the most commonly used doubly rotated cut in a quartz crystal, $1 \mathrm{~mm}$ thick, $18 \mathrm{~mm}$ in diameter, and with a typical radius of curvature close to $250 \mathrm{~mm}$ (see Fig. 1). Bottom: effective mass versus eigenfrequencies, calculated from Eq. (6).

Actually, quality factors for the observed mode orders display a trend to increase for higher longitudinal mode orders (see Fig. 2), which is expected due to the better lateral confinement of the acoustic energy for modes with shorter wavelength in a realistic, imperfect plano-convex geometry. Moreover, typically beyond $200 \mathrm{MHz}$, scattering due the surface roughness leads to a decrease in the Q-factor according to the $f^{-1}$ law.

The expected normalized eigenvector components for the A-mode in $S C$-cut quartz are $\vec{u}=(0.24,0.97,0.07)$, such that the displacement occurs primarily in the longitudinal direction, ${ }^{10}$ along the symmetry axis of the device ( $y$-direction). For simplicity and clarity of the argument, we will subsequently neglect the non-longitudinal components. The normalized standing-wave mechanical displacement in the longitudinal direction at the angular mode frequency $\Omega_{n m p}$ exhibits a Hermite-Gaussian profile ${ }^{11}$ and can be written in the form

$$
\begin{gathered}
u_{n m p}(x, y, z)=e^{-\frac{\alpha_{n} x^{2}+\beta_{n} z^{2}}{2}} H_{m}\left(\sqrt{\alpha_{n}} x\right) H_{p}\left(\sqrt{\beta_{n} z}\right) \sin \left(\frac{n \pi}{t} y\right), \\
n=1,3,5, \ldots, m, p=0,2,4, \ldots,
\end{gathered}
$$

in the Cartesian coordinate system (depending on the cut angles), where $H_{m}$ and $H_{p}$ are Hermite polynomials, $n$ 
denotes the longitudinal mode order (values limited to odd numbers are dictated by the electrical boundary conditions that do not make sense anymore in the case of nonpiezoelectric materials), and $m$ and $p$ transverse orders

$$
\alpha_{n}=n \pi \sqrt{\frac{C_{e}}{R t^{3} M_{n}}}, \quad \beta_{n}=n \pi \sqrt{\frac{C_{e}}{R t^{3} P_{n}}},
$$

with $R$ denotes the radius of curvature, $t$ the cavity thickness at the center, $C_{e}$ the effective stiffness, and $M_{n}, P_{n}$ the dispersion coefficients of the mode considered among the three existing modes, and depending on the selected quartz cut. The corresponding angular eigenfrequencies for the $n$-th longitudinal overtone with the fundamental transverse order, i.e., a Gaussian profile, are given by

$$
\Omega_{n 00}^{2}=\frac{n^{2} \pi^{2} C_{e}}{t^{2} \rho}\left[1+\frac{1}{n \pi} \sqrt{\frac{t}{R}}\left\{\sqrt{\frac{M_{n}}{C_{e}}}+\sqrt{\frac{P_{n}}{C_{e}}}\right\}\right] \approx \frac{n^{2} \pi^{2} C_{e}}{t^{2} \rho},
$$

where $\rho$ is the density. The approximation in (3) is possible in the case of the A-mode of a typical SC-cut quartz cavity with parameters as in Fig. 2, where typical values for highorder trapped overtones are $C_{e} \approx 121 \times 10^{9} \mathrm{~N} \mathrm{~m}^{-2}$ and $M_{n}$ $\approx 40 \times 10^{9} \mathrm{~N} \mathrm{~m}^{-2}, P_{n} \approx 50 \times 10^{9} \mathrm{~N} \mathrm{~m}^{-2}$.

Equation (1) can be rewritten in a more convenient form by using the same notations as for an optical mode in the paraxial approximation as

$$
\begin{aligned}
u_{n m p}(x, y, z)= & e^{-\frac{x^{2} \sqrt{P_{n}}}{w_{n}^{2}}} H_{m}\left(\frac{\sqrt{2} P_{n}^{1 / 4}}{w_{n}} x\right) e^{-\frac{z^{2} \sqrt{M_{n}}}{w_{n}^{2}}} \\
& \times H_{p}\left(\frac{\sqrt{2} M_{n}^{1 / 4}}{w_{n}} z\right) \sin \left(\frac{n \pi}{t} y\right),
\end{aligned}
$$

where $w_{n}^{2}$ is the acoustical waist defined by

$$
w_{n}^{2}=\frac{2 t \sqrt{R t}}{n \pi} \frac{\sqrt{M_{n} P_{n}}}{\sqrt{C_{e}}} .
$$

While these modes can be driven by a moderate electric field along the $y$ axis in piezoelectric materials such as quartz, it may be harder to provide a sufficient force through radiation-pressure in an optomechanical setting. To be able to consider this quantitatively in Sec. III, it is useful to map the three-dimensional shapes of the optical and mechanical modes to a one-dimensional optomechanical toy-model, consisting of a mass on a spring reflecting a laser beam circulating inside a Fabry-Perot cavity. ${ }^{12}$ The effective mass in this model depends on the location of the mechanical mode probed by the laser beam. We will only consider the optimal case of a laser beam propagating along the axis of a BAW resonator that is reflected by its outer surface. The effective mass of a given n00-type overtone, whose waist $w_{n}$ is much larger than the optical beam waist, can then be approximated by integrating (1) or (4) over the cavity volume and multiplying with the density $\rho$

$$
m_{n 00}=\frac{\rho t^{2} \sqrt{R t}}{2 n} \frac{\sqrt[4]{M_{n} P_{n}}}{\sqrt{C_{e}}}=\frac{\rho \pi w_{n}^{2} t}{4 \sqrt[4]{M_{n} P_{n}}} .
$$

The effective mass is inversely proportional to the overtoneorder $n$ because the lateral dimension of the acoustic mode is defined by $w_{n}$, whose scaling with $1 / \sqrt{n}$ can be seen as a consequence of the acoustic wavelength being proportional to $1 / n$, in full analogy to the optical case. Typical effective mass values for longitudinal modes in a $1 \mathrm{~mm}$ thick cavity in a SC-cut quartz crystal lie in the sub-mg range and are shown in Fig. 2.

\section{ACOUSTIC CAVITIES USED AS OPTICAL CAVITIES: FEASIBILITY}

The shape and dimension of usual plano-convex BAW cavities are fully compatible with the requirements of optical Fabry-Perot cavities. The only modification required to convert these mechanical cavities into optical ones is an improvement of the reflectivity of both the flat and convex surfaces in order to trap light within the mechanical mode volume. The low optical absorption of many crystalline materials $^{13}$ and the low surface roughness of the BAW cavities under consideration (typically from $0.3 \mathrm{~nm}$ rms to $30 \mathrm{~nm}$ rms) should enable high finesse $\left(\mathcal{F} \gg 10^{4}\right.$ might ideally be reached) provided high enough reflectivities are achieved.

The simplest way to obtain an optical cavity is to coat metal electrodes on both surfaces of a plano-convex substrate, the center part in Fig. 1, which then act simultaneously as mechanical and optical mirrors. This is actually the most widely used configuration for radio-frequency BAW resonators, where metallic electrodes are commonly made of gold, or sometimes of silver or aluminium that should be passivated. Nevertheless, while the room temperature mechanical quality factor of a gold-coated quartz acoustic cavity is essentially unaltered by the presence of the electrodes, the gold layers have caused a 4-fold reduction of the $Q$-factor at $4 \mathrm{~K}$ in previous experiments. ${ }^{14}$ Typical $150 \mathrm{~nm}$ thick metal electrodes would exhibit reflectivities close to $95 \%-98 \%$ at a wavelength $\lambda=1550 \mathrm{~nm}$, resulting in an expected (acceptable) finesse $\mathcal{F}$ on the order of 100. Preliminary experiments at room temperature have shown that a metal-coated plano-convex acoustic cavity in quartz crystal exhibits optical resonances, but results were not the expected ones. Indeed, gold electrodes used on conventional quartz resonators turn out to be a poor option as optical reflectors. Although such electrodes are basically 100-150 nm thick gold layers, actually they can adhere onto the quartz substrate by means of an additional light veil of chromium. As a consequence, even if the latter is much less than $50 \mathrm{~nm}$ thick, the light supposed to be trapped inside the quartz cavity between both electrodes sees these chromium layers first, i.e., very poor reflectors, instead of gold. Tests have also been carried out without the chromium layers but the formation of small bubbles have been observed: gold alone can undergo delamination. ${ }^{15}$ Silver looks likely to be a more efficient solution: it exhibits a reflectivity similar to, and even better than that of gold in the near-IR range, and in addition can be directly coated onto quartz without an extra underlay. Very first tests with silver coatings demonstrate that the $1 \mathrm{~mm}$ thick planoconvex quartz lens operates well like a Fabry-Perot optical cavity with a measured resonance frequency width just slightly 
lower than $10 \mathrm{GHz}$. This value, larger than expected, might result from the overlay of a set of various modes around the desired one. Parasitic modes are still better to be rejected: the optimization of metal coatings, thickness, and diameter of both mirrors, is currently in progress. ${ }^{16}$ Nonlinearities caused by thermal expansion of the quartz resonator that is triggered by the resonant absorption of light is also an issue to be investigated.

The mirror reflectivity can be improved further by coating Bragg-type mirrors instead of simple metal layers. For example, coatings made of alternating $\lambda / 4$-layers of $\mathrm{SiO}_{2}$ and $\mathrm{Ta}_{2} \mathrm{O}_{5}$ allow for reflectivities greater than $99.99 \%$, and consequently a finesse well above $10^{4}$. However, the mechanical quality factors are lowered even more by these coatings: at $4 \mathrm{~K}$, a $Q$-factor decrease in one to two orders of magnitude has been measured with quartz acoustic cavities. ${ }^{14}$ The high mechanical loss of dielectric coatings is well-known in the community of gravitational-wave interferometers, ${ }^{17}$ and future improvements might arise from the use crystalline mirror coatings ${ }^{18}$ or photonic crystal mirrors. ${ }^{19}$

Figure 3(a) shows the measured reflection spectrum for a BAW cavity made from $\mathrm{CaF}_{2}$ that was coated with highly reflective dielectric mirrors on both sides. With a refractive index of $n=1.42$, the measured optical linewidth translates to a finesse of $\mathcal{F}=135000$ and an optical loss of $\mathcal{L}<40 \mathrm{ppm}$. The achieved very high finesse shows that bulk absorption and surface roughness are indeed low enough to make BAW cavities an attractive alternative to conventional optomechanical systems.

\section{OPTOMECHANICAL CAVITIES: OPTIONS}

Thus, acoustic cavities can definitely be converted into optical cavities starting from the basic solution discussed above, but optimal optomechanical performance requires a tradeoff between mechanical and optical performance (see Fig. 4). To mitigate this tradeoff, a more sophisticated option is to combine a separate dielectric mirror with the planoconvex resonator as shown in Fig. 4(b), which allows coating of only one of the two surfaces with a mirror and achieves optimal optical and mechanical performance on the other surface. While technically even more challenging, the piezoelectric "sandwich"-scheme from Fig. 1 can also be applied to optomechanics: embedding the acoustic cavity inside an optical cavity with two separately mounted dielectric mirrors as shown in Fig. 4(c) fully removes the tradeoff between optics and mechanics and allows for optimal performance in both domains.

The last two options involve one or two uncoated surfaces inside the optical cavity, which might result in significant optical loss. To study the impact of uncoated crystal interfaces, the scheme proposed in Fig. 4(b) has been tested with a 1-mm thick plano-convex lens made of $\mathrm{CaF}_{2}$. The convex surface was coated with a dielectric mirror with transmission and absorption below $1 \mathrm{ppm}$. A separate concave dielectric mirror with a transmission of $100 \mathrm{ppm}$ was placed at a variable distance from the plano-convex mirror to form a FabryPerot cavity surrounding the flat crystal surface. Figure 3(b) shows measurements of the round-trip loss for variable cavity length. The lowest optical loss is expected when the wavefront of the optical beam matches the shape of the uncoated crystal surface (at a length of $7.1 \mathrm{~mm}$ in this case). However, it was impossible to avoid small angular misalignments of the uncoated crystal surface with respect to the cavity axis in this experiment, which is expected to induce additional loss. To study this effect as well, Fig. 3(c) shows a scan of this interface angle. While the data display a broad loss minimum around the expected zero angle, the measured values are significantly scattered. This could be explained by small but unavoidable alterations of the orthogonal interface angle and cavity length during the angular scan.

The separation of the mirror from the mechanical surface in the last two options [as in Figs. 4(b) and 4(c)] also removes the momentum exchange between light and mechanics associated with the reflection by that surface, and should therefore be accompanied by a lower or even zero optomechanical coupling. However, in practice, an appreciable coupling is provided by thermal or photoelastic effects, where the volume overlap integral of mechanical strain and optical intensity defines the coupling strength, opposed to the surface overlap

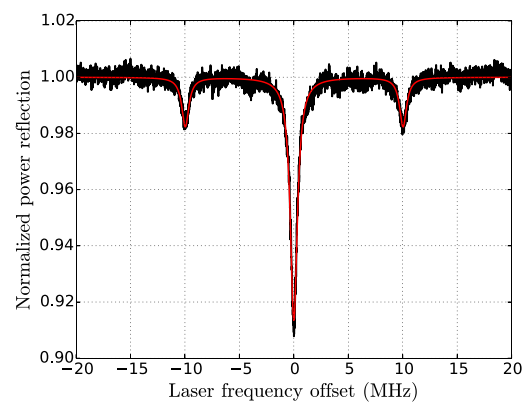

(a)

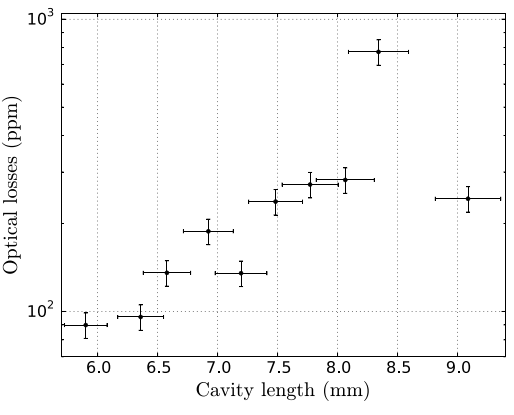

(b)

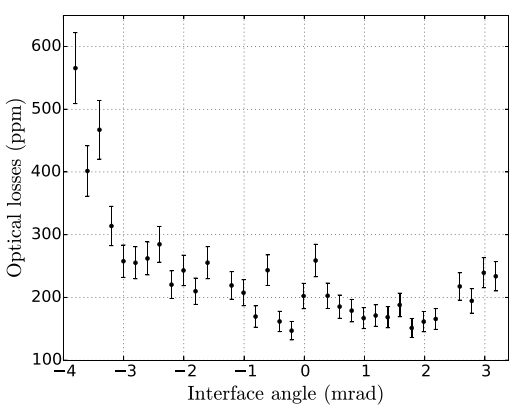

(c)

FIG. 3. (a) Reflection across a Fabry-Perot resonance of a 1-mm thick $\mathrm{CaF}_{2}$ plano-convex lens coated with dielectric Bragg mirrors on both surfaces [geometry as in Fig. 4(a)], operating in air at room temperature. The measurement record (black) displays two phase modulation sidebands $\left(f_{\bmod }=10 \mathrm{MHz}\right)$ which allow to extract the resonance linewidth $\kappa=780 \mathrm{kHz}$ from a Lorenzian fit (red). (b) and (c) Measured cavity round-trip loss for a cavity made from a plano-convex $\mathrm{CaF}_{2}$ crystal with the geometry as in Fig. 4(b) as a function of cavity length (b) and interface angle for a cavity length of $7.1 \mathrm{~mm}$ (c). The independently measured input coupler transmission $(100 \mathrm{ppm})$ was subtracted from the data for better visibility, and the remaining transmissions and absorptions of both mirrors are expected to be negligible (sum $<5 \mathrm{ppm}$ ) with respect to scattering loss due to mirror surface roughness (about $20 \mathrm{ppm}$ ), interface-related scattering, and clipping on an aperture inside the cavity. 

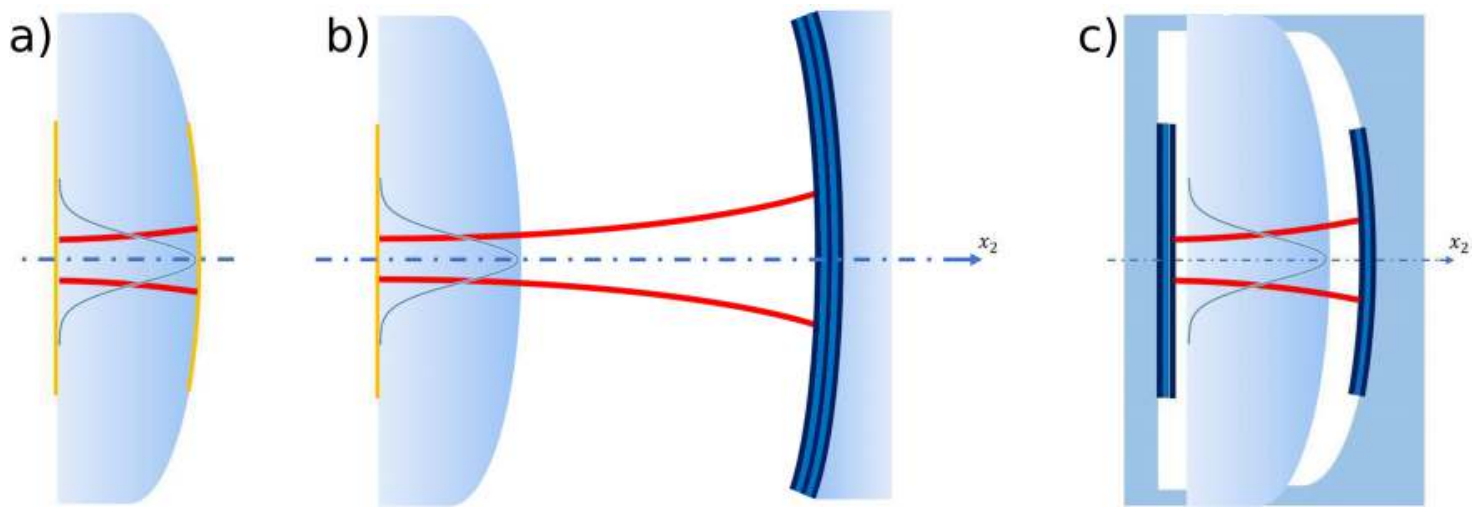

FIG. 4. Different strategies to convert plano-convex BAW devices into optical cavities. The blue line shows the Gaussian distribution of mechanical displacement and the red lines indicated the spatial shape of the laser beam inside the cavity. (a) Acoustic device used as an optical cavity with gold electrodes coated on both surfaces, acting as optical mirrors. (b) Optomechanical system made of a gold-coated plano-convex acoustic cavity combined with a dielectric supermirror. (c) Optomechanical system made of two separate dielectric mirrors on both sides of the acoustic cavity.

integral between mechanical displacement and optical intensity in conventional "moving-boundary" optomechanical coupling. A redefinition of the effective mode mass can be used to link these modified situations to the canonical optomechanical formalism.

\section{OPTOMECHANICAL CAVITIES: ACTUATION AND READOUT}

The laser beam is assumed to be along the y-axis, i.e., along the thickness of the acoustic/optical cavity. The light intensity $I$ being the number of photons per unit of time, limited to its average part in the analysis below, the laser power can be expressed as $P_{\text {laser }}=\frac{h c}{\lambda} I$. Furthermore, when neglecting the influence of the spatial pattern of the electric field at the level of a mirror plane, the radiation-pressure force onto the latter is $F_{r a d}=2 \hbar k I$, where $2 \hbar k$ is the photon impulse transfer ( $k$ is the wave-number). Thus, when a laser beam of power $P_{\text {laser }}$ is incident on the cavity, the circulating intracavity power, for a resonant laser, is given by

$$
P_{\text {cavity }}=P_{\text {laser }} \frac{2 \mathcal{F}}{\pi} \frac{T}{T+P},
$$

when the input "coupler"-mirror is assumed to have a power transmission $T$ and the "loss-port" mirror to lose a fraction $P$ of the power reflected off it. The first fraction in (7) describes the finesse enhancement and the second fraction describes the "impedance matching." In addition, when neglecting photoelastic coupling in the following and considering only the standard optomechanical coupling, i.e., the moving boundaries, the radiation-pressure force from the intracavity beam is then given by

$$
F_{\text {rad }}=\frac{2 P_{\text {cavity }}}{c_{c}},
$$

where $c_{c}$ is the light speed inside the cavity (the speed of light in vacuum $c$ divided by the refractive index of the material).

A cavity interacting with a laser beam is then submitted to the radiation-pressure force in addition to the Langevin force due to random thermal fluctuations according to the fluctuation-dissipation theorem. The latter will not be taken into account. The radiation-pressure force, that includes also a random part coming from the laser, can efficiently be related in the Fourier domain to the mirror displacement $y(t)$ as $\delta y[\Omega]=\chi_{n}[\Omega] \delta F_{\text {rad }}[\Omega]$ for a given mode $n 00$ (denoted $n$ for simplicity), where $\chi_{n}[\Omega]$ is the mechanical susceptibility close to the resonance

$$
\chi_{n}[\Omega]=\frac{1}{m_{n}\left(\Omega_{n}^{2}-\Omega^{2}-i \Omega_{n}^{2} \phi(\omega)\right)},
$$

with $\phi(\omega)=\frac{\Omega}{Q_{n} \Omega_{n}}$ being the mechanical loss of the overtone $n$ (for $n 00$ ) in the case of viscous damping. Thus, from previous equations and with the approximations mentioned above, the relationship between the mechanical displacement and the modulation of the laser power $\delta P_{\text {laser }}[\Omega]$ can be expressed as

$$
\delta y[\Omega]=\frac{4}{c_{c}} \frac{\mathcal{F}}{\pi} \frac{T}{T+P} \chi_{n}[\Omega] \delta P_{\text {laser }}[\Omega],
$$

which yields at the mechanical resonance angular frequency $\Omega_{n}$, when divided by 2 , it yields the effective mass because the optical beam probes the mechanical displacement twice per round-trip in the cavity, but assuming that $T \approx P$

$$
\left|\frac{\delta y\left[\Omega_{n}\right]}{\delta P_{\text {laser }}}\right| \approx \frac{4 \mathcal{F}}{\pi c_{c}} \frac{Q_{n}}{m_{n} \Omega_{n}^{2}}=K_{n} \frac{Q_{n}}{n},
$$

with $K_{n}=\frac{2 \mathcal{F}}{\pi^{3} c_{c} \sqrt{R} t} \frac{1}{M_{n}^{1 / 4} P_{n}^{1 / 4} C_{e}^{1 / 2}}$. Thus, the displacement sensitivity to laser power is proportional to the ratio $Q_{n} / n$. The sensitivity calculated by means of (11) from data shown above (see Fig. 2) is illustrated in Fig. 5, for a finesse $\mathcal{F}=80$. As a result, an acoustic quartz cavity simply metal coated, would exhibit displacement values on the order of $1.310^{-10} \mathrm{~m}$ per $\mathrm{mW}$ of actuating laser modulation.

Furthermore, the detection noise floor is given by the shot noise of the laser beam and can be estimated from ${ }^{4}$

$$
\delta y_{\text {shot }}[\Omega]=\frac{1}{16 \mathcal{F}} \sqrt{\frac{2 h c \lambda}{P_{\text {laser }}}} .
$$

This yields to a displacement noise floor, assuming for example a perfect homodyne detection for a laser resonant with an 



FIG. 5. Top: $Q_{n} / n$ ratio versus acoustic-resonance frequencies of $n^{\text {th }}$ overtones at $4 \mathrm{~K}$ for the resonators from Fig. 2. Bottom: expected cavity length change per unit of modulated laser power versus acoustic-resonance frequencies, calculated from Eq. (11) for a given finesse $\mathcal{F}=80$, a minimum value assessed with gold or silver mirrors. For comparison, the noise floor of a shot-noise limited detector in a $1 \mathrm{~Hz}$ detection band and $1 \mathrm{~W}$ laser power is expected at $6 \times 10^{-19} \mathrm{~m}$, about 10 orders of magnitude below the expected cavity length change signals shown here [see Eq. (12) and the main text].

optical cavity mode, of $2 \times 10^{-17} \mathrm{~m} / \sqrt{\mathrm{Hz}}$ for a $1 \mathrm{~mW}$ laser power at $1550 \mathrm{~nm}$. One of the main motivations of using mechanical modes with ultrahigh mechanical quality factors is to keep thermal displacement noise, which is related to the mechanical quality factor via the Fluctuation-DissipationTheorem, as low as possible to achieve maximum coherence for quantum state preparation and measurement. It is wellknown in the community of optomechanics that optomechanical cooling cannot improve the signal-to-thermal-noise ratio, but only trades a lower effective temperature for lower effective mechanical quality factor. Therefore, the solution for keeping thermal noise low is to use mechanical resonators with the highest mechanical quality factors that are available. Actually, the previous calculation, with $T \approx P$ and a finesse $\mathcal{F}=80$, has neglected optical loss and other technical limitations when probing the displacement as space matching of the beam with the cavity, the filter effect of the cavity, and the photodiode efficiency. So, this assessment should likely be increased but nevertheless shows that probing the displacement of the cavity actuated optically is realistic.

\section{PROGRESS AND CONCLUSION}

Very promising preliminary tests have been carried out on quartz as well as $\mathrm{CaF} 2$ plano-convex bulk cavities and alternative solutions discussed. Although optically excitation is theoretically available, issues still arise to know how photoelastic and thermal effects for example are involved in the interaction process of optics to and from mechanics, in comparison with radiation effects. While the options discussed above are going to be investigated, studies on quartz temperature-compensated cuts are also in progress in parallel. Indeed, since the elastic coefficients, density, and thickness of the acoustic cavity depend on temperature, the acoustic-resonance frequency is also temperature dependent, and it becomes mandatory to select the appropriate cut angles exhibiting a frequency-temperature turning point in the $[3 \mathrm{~K}-15 \mathrm{~K}]$ range. The temperature coefficients of the stiffness coefficients have recently been measured ${ }^{20}$ and frequency-temperature compensated cuts have then been identified: devices are currently under test to check the angle sensitivity.

\section{ACKNOWLEDGMENTS}

Thanks to Xavier Vacheret for his help in making resonators, Jacques Millo and Clément Lacroûte for their help on the experimental set up design, Hoang-Van Do for help with some measurements, Khaldoun Saleh for his help on the laser properties' characterization, and Ludovic Gauthier-Manuel and Thibaut Sylvestre for their help on the welding of AOM connectors. This work is supported by the Conseil Régional de Franche-Comté and Labex FIRST-TF. J.B. is thankful to the Ministère de l'Enseignement Supérieur et de la Recherche, France, for their grant. We also thank the platform Oscillator-Imp for the distribution of a reference hydrogen-maser signal in the laboratory.

${ }^{1}$ S. Galliou, J. Imbaud, M. Goryachev, R. Bourquin, and P. Abbé, "Losses in high quality quartz crystal resonators at cryogenic temperatures," Appl. Phys. Lett. 98, 091911 (2011).

${ }^{2}$ S. Galliou, M. Goryachev, R. Bourquin, P. Abbé, J. P. Aubry, and M. E. Tobar, "Extremely low loss phonon-trapping cryogenic acoustic cavities for future physical experiments," Sci. Rep. 3, 2132 (2013).

${ }^{3}$ M. Aspelmeyer, T. J. Kippenberg, and F. Marquardt, "Cavity optomechanics," Rev. Mod. Phys. 86, 1391 (2014).

${ }^{4} \mathrm{~T}$. Briant, "Caractérisation du couplage optomécanique entre la lumiére et un miroir: Bruit thermique et effets quantiques," Ph.D. thesis (Université Pierre et Marie Curie, Paris VI, 2004).

${ }^{5}$ A. Lo, P. Haslinger, E. Mizrachi, L. Anderegg, H. Müller, M. Hohensee, M. Goryachev, and M. E. Tobar, "Acoustic tests of Lorentz symmetry using quartz oscillators,” Phys. Rev. X 6, 011018 (2016).

${ }^{6}$ S. Kotler, R. W. Simmonds, D. Leibfried, and D. J. Wineland, "Hybrid quantum systems with trapped charged particles," Phys. Rev. A 95, 022327 (2017).

${ }^{7}$ A. Akheiser, "On the absorption of sound in solids," J. Phys.-USSR 1, 277 (1939).

${ }^{8}$ L. Landau and G. Rumer, "Uber schall absorption in festen Körpen," Phys. Z. Sowjetunion 11, 18-25 (1937).

${ }^{9} \mathrm{H}$. Maris, "Interaction of sound waves with thermal phonons in dielectric crystals," in Physical Acoustics (Academic, 1971), pp. 279-345.

${ }^{10}$ D.-A. Nassour, "Influence des paramètres de construction sur le défaut d'isochronisme des résonateurs piézoélectriques à simple ou double rotation," thesis es Sciences Physiques (University of Franche Comté, France, 1982). 
${ }^{11}$ D. S. Stevens and H. F. Tiersten, "An analysis of doubly rotated quartz resonators utilizing essentially thickness modes with transverse variation," J. Acoust. Soc. Am. 79, 1811 (1986).

${ }^{12}$ M. Pinard, Y. Hadjar, and A. Heidmann, "Effective mass in quantum effects of radiation pressure," Eur. Phys. J. D 7, 107 (1999).

${ }^{13}$ A. A. Savchenkov, V. S. Ilchenko, A. B. Matsko, and L. Maleki, "Kilohertz optical resonances in dielectric crystal cavities," Phys. Rev. A 70, 051804 (2004).

${ }^{14}$ S. Galliou, S. Deléglise, M. Goryachev, L. Neuhaus, G. Cagnoli, S. Zerkani, V. Dolique, J. Bon, X. Vacheret, P. Abbé, L. Pinard, C. Michel, T. Karassouloff, T. Briant, P.-F. Cohadon, A. Heidmann, M. E. Tobar, and R. Bourquin, "A new method of probing mechanical losses of coatings at cryogenic temperatures," Rev. Sci. Instrum. 87, 123906 (2016).

${ }^{15}$ N. B. Dahmane, G. Parry, and R. Estevez, "Cloquage de films minces ductiles sur substrat rigide," in 22ème Congrès Français de Mécanique, Lyon (2015).
${ }^{16}$ J. J. Monzòn and L. L. Sànchez-Soto, "On the concept of absorption for a Fabry-Perot interferometer,” Am. J. Phys. 64, 156-163 (1996).

${ }^{17}$ E. Hirose, K. Craig, H. Ishitsuka, I. W. Martin, N. Mio, S. Moriwaki, P. G. Murray, M. Ohashi, S. Rowan, Y. Sakakibara, T. Suzuki, K. Waseda, K. Watanabe, and K. Yamamoto, "Mechanical loss of a multilayer tantala/ silica coating on a sapphire disk at cryogenic temperatures: Toward the kagra gravitational wave detector," Phys. Rev. D 90, 102004 (2014).

${ }^{18}$ G. Cole, W. Zhang, M. Martin, J. Ye, and M. Aspelmeyer, "Tenfold reduction of Brownian noise in high-reflectivity optical coatings," Nat. Photonics 7, 644 (2013).

${ }^{19}$ F. Brückner, D. Friedrich, T. Clausnitzer, M. Britzger, O. Burmeister, K. Danzmann, E.-B. Kley, A. Tünnermann, and R. Schnabel, "Realization of a monolithic high-reflectivity cavity mirror from a single silicon crystal," Phys. Rev. Lett. 104, 163903 (2010).

${ }^{20} \mathrm{~J}$. Bon, S. Galliou, and R. Bourquin, "Temperature coefficients of crystalline-quartz elastic constants over the cryogenic range [4 k, $15 \mathrm{k}]$," Appl. Phys. Lett. 109, 231902 (2016). 